\title{
Carnets
}

Revue électronique d'études françaises de l'APEF

Première Série - 3 Numéro Spécial | 2011

La littérature face au "politiquement correct». Notions, pratiques et dérives

\section{Politiquement correct: tour d'horizon et acceptions}

\section{Nicolina Almeida}

\section{CpenEdition}

Journals

Édition électronique

URL : http://journals.openedition.org/carnets/6304

DOI : $10.4000 /$ carnets. 6304

ISSN : 1646-7698

Éditeur

APEF

Édition imprimée

Date de publication : 1 juin 2011

Pagination : 19-25

Référence électronique

Nicolina Almeida, "Politiquement correct: tour d'horizon et acceptions », Carnets [En ligne], Première Série - 3 Numéro Spécial | 2011, mis en ligne le 19 juin 2018, consulté le 19 avril 2019. URL : http:// journals.openedition.org/carnets/6304 ; DOI : 10.4000/carnets.6304

\section{(c) (i) ()ㅜㄹ}

Carnets est mis à disposition selon les termes de la licence Creative Commons - Atribution - Pas d'utilisation commerciale 4.0 International. 


\title{
POLITIQUEMENT CORRECT: TOUR D'HORIZON ET ACCEPTIONS ${ }^{1}$
}

Nicolina ALMEIDA

Faculté des Lettres de l'Université de Porto

nicolealmeida@hotmail.fr

\begin{abstract}
Résumé
Du Political Correctness américain à nos jours le code de bonne conduite est de rigueur. L'auteur aborde le politiquement correct sous différentes perspectives allant de la politique à I'humour en passant par la déclaration des Droits de l'Homme. Une vision large du concept qui n'omet pas de s'interroger sur les acceptions d'une expression bien souvent assimilée à l'hypocrisie ou encore à la langue de bois et qui conclu par un constat: la grande capacité d'adaptation de l'homme lui permettant de toujours développer de nouvelles stratégies oratoires.
\end{abstract}

\section{Abstract}

Ever since the rise of Political Correctness in America, the "code of good practice" is the proper procedure. The author approaches political correctness from different perspectives, from politics to the Declaration of the Rights of Man to humour. A general view of the concept is presented which includes a look into the meaning of the expression, very often associated with hypocrisy or with the "Langue de bois", leading to the conclusion that it is man's great capacity for adaptation that allows him to continuously develop new oratory strategies.

Mots-clés: politiquement correct, politique, humour, identité, nation

Keywords: political correctness, politics, humour, identity, nation

\footnotetext{
${ }^{1}$ Cette communication a été élaborée dans le cadre des "Rendez-vous de la Critique 2010" - La littérature face au "politiquement correct" de la Faculté des Lettres de l'Université de Porto.
} 
Depuis le political correctness américain des années 70 et 80 imposant un code de langage aux locuteurs, le politiquement correct s'insère dans le discours bien pensant et convenable. Plus personne ne se risque à qualifier de "chômeur" un demandeur d'emploi, de "nain" une personne de petite taille ou encore de "vieillesse" le troisième âge. De fait, il est difficile de nos jours d'évoquer certains concepts et idées, alors bien souvent taxés de "sujets délicats", sans craindre d'encourir un politiquement incorrect que d'aucuns vont jusqu'à qualifier de "milice du langage" voire de "puritanisme verbal"

Eric Zemmour est bien placé pour le dire. En effet, l'écrivain et journaliste politique français a fait les frais de son outrecuidance lors d'une émission diffusée sur Canal+ en mars dernier. La Ligue Contre le Racisme et l'Antisémitisme (LICRA) a poursuivit Éric Zemmour en justice pour ses propos dans l'émission Salut les Terriens présentée par Thierry Ardisson dans laquelle il faisait la promotion de son livre Mélancolie française. II a déclaré que: "les Français issus de l'immigration étaient plus contrôlés que les autres parce que la plupart des trafiquants sont noirs et arabes... c'est un fait ${ }^{3 \prime}$. A la suite des poursuites engagées par la LICRA, de nombreuses associations telles que le Club Averroès et le Mouvement contre le Racisme et pour l'Amitié entre les Peuples (MRAP) ont également saisi le Conseil Supérieur de l'Audiovisuel (CSA).

Ce cas concret illustre bien à quel point les propos tenus engagent toujours leur auteur et à plus forte raison dans un contexte médiatique qui, de part sa grande exposition, peut le conduire devant les tribunaux. Selon le philosophe Bertrand Vergely s'est donc mis en place "un mécanisme de terreur sociale" (apud, Castets: 2010) qui ne se contente pas "d'exclure les réfractaires au parler correct" (idem, ibidem) mais les oblige à rendre des comptes. Dans un tel contexte se pose alors légitimement la question de la liberté d'expression.

L'être humain constitué d'un ensemble de différences et de préférences se voit confronté à l'impossibilité d'affirmer sa pensée haut et fort sous peine d'un "abattage" médiatique pour ceux qui s'exposent, voire d'un lynchage en règle infligé par la Société qui contrôle toute la recevabilité de leurs propos. II ne s'agit donc pas seulement d'exprimer une opinion mais également de la justifier sous peine de représailles. L'affrontement d'idées, le débat, deviennent alors les meilleurs moyens de désamorcer la situation dès lors que la Société est disposée à créer un espace de discussion et de liberté de ton inconditionnel.

Mais faut-il être une femme pour parler des femmes, juif pour s'exprimer sur les juifs ou encore séropositif pour évoquer la maladie? Dans quelle mesure peut-on combattre la

\footnotetext{
${ }^{2}$ V. Castets Caroline; Le Nouvel Economiste.fr (2010) - Les dévoiements du politiquement correct. Le site: <URL: http://www.lenouveleconomiste.fr/we-have-a-dream/>. (Consulté le 29/11/10).

3 V. Contrepoints (2010) - Etat de la liberté d'expression en France. Le site: <URL: http://archives.contrepoints.org/Etat-de-la-liberte-d-expression-en.html>. (Consulté le 29/11/10).
} 
discrimination, le préjugé sans les nommer? Rappelons qu'Albert Camus disait: "mal nommer les choses, c'est ajouter aux malheurs du monde."

La Déclaration des droits de l'homme et du citoyen de 1789 constate la liberté d'expression dans son article 11: “(...) tout citoyen peut parler, écrire, imprimer librement $(\ldots)^{4 "}$ mais la Convention européenne des droits de l'homme de nos jours nous dit aussi: “(...) l'exercice de ces libertés peut être soumis à certaines formalités, conditions, restrictions ou sanctions prévues par la loi $(\ldots)^{5 "}$. Alors qu'en est-il de cette liberté d'expression? Est-elle limitée par la liberté de chacun, par les devoirs et responsabilités envers les autres ou bien pouvoir tout exprimer est-il un droit fondamental qui ne doit s'astreindre à aucune censure? Quelles frontières séparent le politiquement correct de l'euphémisme ou encore la langue de bois de l'hypocrisie? L'objectivité est-elle mise à mal par le politiquement correct? Autant de questions qui nous invitent à mener une réflexion, un débat pour lequel il nous convient de décider s'il doit rester politiquement correct ou non.

Telle est la question que doivent se poser de nombreux hommes politiques: jusqu'où puis-je aller? Quel est ce point, difficilement définissable, qui lorsqu'il est atteint n'offre pas de possibilité de retour? II ne s'agit pas seulement de ce que l'on peut se permettre de dire mais également de ce que l'on est autorisé à faire sans oublier que tout dépend de celui qui écoute et observe. Et les citoyens français observent de loin les agissements de leurs hommes politiques. Héritiers d'une longue tradition de "favorites", ces derniers sont reconnus pour leurs "liaisons dangereuses".

Au cours d'une entrevue avec les auteurs de Sexus Politicus, un ouvrage polémique sur la vie privée des hommes politiques français, l'avocat et ministre Patrick Devedjean affirme que les députés, les ministres voire les présidents de la République française succèdent à Henri IV, Louis XIV, Napoléon dans les institutions issues "de la décadence romaine" contrairement à celles des américains créées par les descendants puritains du Mayflower.

Dans cet ouvrage, les auteurs Christophe Deloire et Christophe Dubois citent l'exemple de François Mitterrand qui réussit à garder le secret de sa double vie bénéficiant de la bienveillance de la presse et vont jusqu'à affirmer que:

"Nos présidents n'auraient pas le respect de leurs électeurs s'ils se comportaient d'une autre façon'." (Deloire/Dubois: 2008, 181) II est vrai que le "Monicagate" de Bill Clinton n'a pas choqué la France mais la fait plutôt rire... II est vrai aussi qu'Outre-Atlantique la liberté sexuelle des dirigeants français semble être une légende...

\footnotetext{
${ }^{4}$ V. L'Assemblée Nationale (2010) - La Déclaration des droits de l'homme et du citoyen de 1789. Le site: <URL: http://www.assemblee-nationale.fr/histoire/dudh/1789.asp>. (Consulté le 29/11/10).

5 V. le Conseil de l'Europe (2010) - Convention de sauvegarde des Droits de l'Homme et des Libertés fondamentales. Le site <URL: http://conventions.coe.int/treaty/fr/Summaries/Html/005.htm>. (Consulté le 29/11/10).

${ }^{6}$ C'est nous qui traduisons.
} 
Plus sérieusement, lorsque des voix discordantes s'élèvent telle que celle, toujours véhémente, de la vice-présidente du Front National se prononçant pour la suppression de la double nationalité, lorsqu'elle pointe du doigt Nicolas Sarkozy l'accusant de lui voler ses électeurs avec la proposition de suppression de la nationalité française à quiconque agresserait la force publique... C'est ce que l'on pourrait qualifier aussi d'une attitude politiquement incorrecte mais dans ce cas ne pourrait-on pas admettre que cela fait partie de ses fonctions et du jeu politique? En effet, heurter l'opinion publique, la pousser dans ses retranchements et donc à prendre des décisions radicales lors de son passage aux urnes est l'une des stratégies de tous les partis de l'opposition et en particulier du Front National. En définitive, toucher à des concepts tels que ceux de la nation, de l'identité, de la culture et d'autres sujets sensibles pour l'opinion publique, conduit à une prise de risque que courent bien des hommes politiques, celle de ne pas être politiquement correct tout en évitant de tomber dans l'hypocrisie.

Pensons également aux humoristes dont pour certains la principale fonction est de flirter avec le politiquement correct leur cible de prédilection étant les politiques. Des hommes tels que Pierre Desproges et Guy Bedos, pour n'évoquer que les plus polémiques, en on fait leur spécialité. Alors que le premier laisse perplexe en visionnant son sketch "on me dit que des juifs se sont glissés dans la salle"” voire honteux au moindre sourire esquissé, le second en mars dernier à l'occasion d'un spectacle truculent à Bobino, se fend d'un explicite bras d'honneur à l'encontre d'Eric Besson, le ministre de l'immigration. Alors que Dominique Strauss Khan, "victime" de Patrick Timsit, affirme que: "L'humour s'arrête là où la méchanceté commence ${ }^{8 ",}$, tous s'accordent à dire que le métier d'humoriste a changé depuis mai 2007, date qui coïncide avec l'élection de Nicolas Sarkozy.

L'interdiction le 14 septembre du port du voile intégral sur le sol national et l'expulsion des Roms n'aura pas arrangé les choses. Ces décisions auront terni l'image de la France dans le monde entier et la Commissaire Européenne, Viviane Reding, y aura à sa façon contribué.

Ce que le philosophe Bertrand Vergely appelle le "souci de l'image" aurait-il poussé cette dernière à ce discours moralisateur envers la France? Etait-ce "la peur de l'étiquette qui pousse à jouer la carte de l'indignation permanente, d'autant plus volontiers que celle-ci reste vendeuse"? (idem, ibidem) Cette dernière, pour le coup, dépasse les limites du politiquement correct dans la formulation de ses propos en employant le terme "déportation"

\footnotetext{
${ }^{7}$ Lors de la rédaction de cet article, l'auteur a pu visionner le sketch sur l'Internet. Au moment de sa publication la diffusion de la vidéo avait déjà été interdite sous couvert de la mention: "Bloqué pour des raisons de droits d'auteurs."

${ }^{8}$ V. France 24 (2010) - A Bobino, festival de provocations d'humoristes contre le "politiquement correct". Le site <URL:http://www.france24.com/fr/20100329-a-bobino-festival-provocations-dhumoristes-contre-le-politiquementcorrect>. (Consulté le 01/10/10).
} 
mais pas dans son attitude lorsqu'elle se pose en défenderesse de l'opprimé critiquant ouvertement la politique du gouvernement français.

En effet, porter une accusation de discrimination raciale, émettre un jugement de valeur, c'est déjà stigmatiser et discriminer. On déplore, on désigne des attitudes impropres, tels sont les rapports politiques dans un monde communautaire auquel il faut rendre des comptes. Et c'est le cas de la France qui, traversant une crise identitaire, questionne la nationalité française et interroge les citoyens: "Qu'est-ce qu'être français?". Jean-François Khan, dans son Dictionnaire incorrect (2005), justement, nous livre sa propre définition:

Français. Plus de 80\% des Français le sont "par inadvertance". Même Jospin, même Chirac. L'un et l'autre ont-ils choisi d'être français? Non, bien sûr. Ça s'est trouvé comme ça, par hasard, par la naissance: c'est-à-dire par le sol et par le sang. Querelle absurde, au fond que celle qui oppose les tenants des deux définitions de notre nationalité: car les plus "français", finalement, ne le sont ni par le sol - quoi qu'en pense la gauche -, ni par le sang - quoi qu'en prétende la droite -, ni même par la manifestation de la volonté (quoi qu'elle ne fasse pas de mal); mais par l'engagement. (...) Etre français, c'est peut-être tout simplement avoir apporté quelque chose à la France. (Khan, 2005: 283)

C'est bien là que le bât blesse. Les codes de bonne ou de mauvaise conduite diffèrent selon les partis politiques et Brice Hortefeux a son avis sur la question. Lorsqu'il résiste à la pression médiatique et lance une lutte ouverte à "un totalitarisme sexiste et violent", celui de la polygamie en France en écrivant au journal le Monde: "Je préfère déplaire au Monde que d'empêcher, par mon silence, la manifestation de la vérité, quelle qu'elle soit", il se soumet non seulement à l'opprobre de ce journal qui a suggéré sa "déchéance ministérielle", mais aussi à celle de tous les médias et résiste à un discours, une attitude politiquement corrects que ne manqueront pas de louer les partisans de la laïcité, opposés au port du voile.

Mais revenons à la définition de l'expression "politiquement correct". N'y a-t-il pas ici une redondance? "Politiquement" selon le Centre National de Ressources Textuelles et Lexicales (CNRTL) c'est déjà: "agir avec habileté, avec à propos". De même, selon le Trésor de la Langue Française Informatisé (TLFI) c'est agir "d'une manière fine, adroite, calculée, prudente." La notion de "correction" est-elle vraiment nécessaire ou renforcer l'idée est-il l'expression d'un inconfort devant une notion par trop dérangeante?

Soulignons que les Québécois, toujours engagés et méticuleux lorsqu'il s'agit de défendre de langue française, ne se sont pas contentés d'une simple traduction mot à mot

9 V. Le Jdd.fr (2010) - Hortefeux "préfère déplaire" au Monde - le site <URL: http://www.lejdd.fr/Politique/Depeches/Hortefeux-prefere-deplaire-au-Monde-189348/>. (Consulté le 01/10/10). 
de l'anglais politically correct, ils lui ont choisi l'expression "rectitude politique" qui serait un équivalent plus "linguistiquement correct". Déjà, la "langue de bois" avait recours à l'euphémisation, le "politiquement correct", lui, semble se rassurer à coup d'adjectifs.

Aujourd'hui, on décline de nombreuses locutions dérivées de cette expression: "culturellement -, socialement -, sexuellement correct"... Sans oublier le: "grammaticalement correct" bien sûr. La définition s'est donc élargie. Selon le Larousse.fr: "le politiquement correct, se dit d'un discours, d'un comportement visant à bannir tout ce qui pourrait blesser les membres de catégories ou de groupes minoritaires en leur faisant sentir leur différence comme une infériorité ou un motif d'exclusion." A ne pas confondre avec la langue de bois.

En effet, selon Gérald Antoine, de l'Académie des sciences morales et politiques ${ }^{10}$, ce parent proche du "politiquement correct" comporte tout de même une nuance: le vrai sens du discours est renforcé à l'aide de l'euphémisme, du lieu commun et autre phraséologie mais n'est compris que d'une poignée d'initiés. C'est ainsi que, par exemple, ces derniers sauront décrypter la terminologie employée dans les domaines de l'économie, de la politique ou encore de l'entreprise.

Le "travailleur involontairement privé d'emploi" apparaîtra alors clairement comme un "chômeur victime d'un licenciement", tout comme "l'aménagement du territoire" reviendra simplement et surtout malheureusement à la "destruction du paysage existant". Le "miracle économique" comme l'interprète Noam Chomsky dans son ouvrage L'An 501, la conquête continue (2006), ne sera autre que: “(..) un ensemble intégré de belles statistiques macroéconomiques, de grands profits pour les investisseurs étrangers et de vie de luxe pour les élites locales; avec, en petits caractères, un accroissement de la misère pour la majorité de la population ${ }^{11}$."

En définitive, l'on peut avancer que la grande maîtrise de la rhétorique propre aux Français, l'éloquence des hommes politiques dans leurs discours maniant l'art de bien dire avant même de persuader, en font des spécialistes du "parler correct". Le pouvoir des mots, de la parole, don précieux qui distinguait les héros grecs est toujours d'actualité, la rhétorique s'adaptant toujours aux nouvelles nécessités. Tant que l'homme aura la faculté de découvrir, de voir, d'observer ce qui l'entoure et de s'accommoder aux circonstances il développera de nouvelles stratégies oratoires.

\footnotetext{
${ }^{10}$ V. Gerald, Antoine (2010) - Académie des sciences morales et politiques - De la "langue de bois" au "politiquement correct" -

Le site: <URL: http://www.asmp.fr/fiches_academiciens/textacad/antoine/languedebois.pdf>. (Consulté le $01 / 10 / 10)$.

${ }^{11}$ V. La Toupie (2010). Langue de bois: quelques exemples en politique, économie, en entreprise... Le site: <URL: http://www.toupie.org/Textes/Langue_de_bois_5.htm>. (Consulté le 1/10/10).
} 


\section{Bibliographie}

DeloIRE, Christophe; Dubois, Christophe (2008). Sexus politicus. Porto: Ambar.

KHAN, Jean-François (2005). Dictionnaire Incorrect. Paris: Plon.

\section{Webographie:}

CASTETS, Caroline (2010). "Les dévoiements du politiquement correct". Le Nouvel Economiste.Fr [en ligne]. [Site consulté le 29 novembre 2010]. <URL: http://www.lenouveleconomiste.fr/we-have-adream/>.

CNRTL (2010). Centre National de Ressources Textuelles et Lexicales [en ligne]. [Site consulté le 15 octobre 2010]. <URL: http://www.cnrtl.fr/definition/politiquement>.

WAGNER, Anton. (16 mars 2010), "Etat de la liberté d'expression en France". Contrepoints [en ligne]. [Site consulté le 29 novembre 2010]. <URL: http://archives.contrepoints.org/Etat-de-la-liberte-dexpression-en.html>.

CSA. (21 avril 2010), "Décisions du CSA - Propos d'Éric Zemmour sur France Ô: le Conseil répond au CRAN". Conseil supérieur de l'audiovisuel [en ligne]. [Site consulté le 15 octobre 2010]. <URL: http://www.csa.fr/actualite/decisions/decisions_detail.php?id=130951>.

AFP. (29 mars 2010), "A Bobino, festival de provocations d'humoristes contre le politiquement correct". France 24 [en ligne]. [Site consulté le 01 octobre 2010]. <URL:

http://www.france24.com/fr/20100329-a-bobino-festival-provocations-dhumoristes-contre-lepolitiquement-correct>.

GERALD, Antoine (2010). "De la 'langue de bois' au 'politiquement correct". Académie des Sciences Morales et Politiques [en ligne]. [Site consulté le 01 octobre 2010]. <URL: http://www.asmp.fr/fiches_academiciens/textacad/antoine/languedebois.pdf>.

LE JDD.FR. (28 avril 2010), "Hortefeux 'préfère déplaire' au Monde". Le Journal du dimanche [en ligne]. [Site consulté le 01 octobre 2010]. <URL: http://www.lejdd.fr/Politique/Depeches/Hortefeux-preferedeplaire-au-Monde-189348/>.

L'Assemblee Nationale (2010). "La Déclaration des droits de l'homme et du citoyen de 1789". [Site consulté le 29 novembre 2010]. <URL: http://www.assemblee-nationale.fr/histoire/dudh/1789.asp>.

LAROUSSE.FR (2010). "Les expressions avec politiquement". [Site consulté le 15 octobre 2010]. <URL: http://www.larousse.fr/dictionnaires/francais/politiquement/62195/locution>.

LA TOUPIE (2010). "Langue de bois: quelques exemples en politique, économie, en entreprise...". [Site consulté le 01 octobre 2010]. <URL: http://www.toupie.org/Textes/Langue_de_bois_5.htm>.

LE CONSEIL DE L'EUROPE (2010). "Convention de sauvegarde des Droits de l'Homme et des Libertés fondamentales". [Site consulté le 29 novembre 2010]. <URL:

http://conventions.coe.int/treaty/fr/Summaries/Html/005.htm>.

LICRA (2010). "La République requiert la condamnation d'Eric Zemmour". [Site consulté le 15 janvier 2011]. <URL: http://www.licra.org/fr/communique/r\%C3\%A9publique-requiert-condamnation-dericzemmour>.

AFP. (11 mars 2010), "Zemmour accusé de racisme: après le Mrap, le club Averroès saisit le CSA". Le Mouvement contre le Racisme et pour l'Amitié entre les Peuples [en ligne]. [Site consulté le 15 octobre 2010]. <URL: http://bonjour.news352.lu/edito-20956-zemmour-accuse-de-racisme-apresle-mrap-le-club-averroes-saisit-le-csa.html>.

TLFI (2010). "Trésor de le Langue Française Informatisé". [Site consulté le 15 octobre 2010]. <URL: http://atilf.atilf.fr/dendien/scripts/generic/cherche.exe?15;s=1554208425>. 
